\title{
Analysis of double-parallel amplified recirculating optical-delay lines
}

\author{
M. C. Vazquez, R. Civera, M. López-Amo, and M. A. Muriel
}

\begin{abstract}
A novel method of analysis of double-parallel amplified recirculating optical-delay lines (DPAROD) is presented. The location of the maxima and the minima of the transfer function for this configuration is calculated and experimentally demonstrated. The influence of different parameters, such as the coupling coefficients, gains, lengths of the fiber loops and fractional losses of the directional couplers, on the shape of the transfer function are analyzed. Different measurements have been taken to verify this model. The potential application of these interconnected delay loops as filters is a reason for developing this method.
\end{abstract}

\section{Introduction}

Single, amplified recirculating optical-delay lines have been widely analyzed, ${ }^{1,2}$ showing their capability for the development of signal-processing functions such as filtering, pulse-train generation, convolution, ${ }^{3}$ and memory. ${ }^{4}$ A higher degree of design flexibility can be achieved with more complex structures, such as the double-parallel amplified recirculating opticaldelay line (DPAROD), ${ }^{1}$ which is depicted in Fig. 1.

New features obtained with DPAROD allow one to reshape their frequency response. For instance, one can obtain a predetermined relative amplitude between the main and secondary maxima and minima of the frequency response of DPAROD. This performance may be fairly useful for filtering, when discrimination is made on the basis not only of the signal frequency, but also on its signal-to-noise ratio. Another application of the DPAROD may be in the field of fiber-optic sensors, owing to the dependence of the DPAROD response on the gain of the fiber loops. If the results to be shown are extrapolated to integrated optics, large free spectral ranges should be achieved with DPAROD, as compared with singlestructure configurations. Previous reports have been made in the coherent analysis of a three-coupler configuration, ${ }^{5}$ while with our DPAROD only two couplers are needed.

An incoherent analysis (i.e., we consider intensities rather than fields) of the DPAROD has been carried out. Our main interest lies in predicting the frequency-domain location of the maxima-minima and the shape of the transfer function, we have developed a new model to do so. Some experimental results are also shown.

\section{Novel Method for Analysis of a Double-Amplified Recirculating Optical-Delay Line}

To describe the signal in fiber-optic recirculating delay lines one usually utilizes the $Z$ transform. ${ }^{6}$ This usage is possible as long as a basic time delay $T$ can be defined such that the other relevant system delays are integer multiples of this basic delay, and the system can be considered discrete in time. Such is the case in the single-structure configuration, ${ }^{1,2}$ or in $N$ th-order fiber-optic lattice structures, where the delay $T$ is the same for all sections. ${ }^{6}$ This is not true for the general DPAROD analyzed in this paper: different loop lengths, the use of which implies different time delays, are used. These time delays do not necessarily have to be integer multiples of a basic time delay, so the $Z$ transform cannot be applied. A novel method will be used.

The DPAROD is a linear system with respect to intensity, so the calculation of the transfer function $H(\Omega)$ (with $I_{1}{ }^{+}$as the input and $I_{2}{ }^{+}$as the output, see Fig. 1) is carried out with the intensity equations of the directional couplers and the recirculating conditions imposed by each loop. The following expres- 


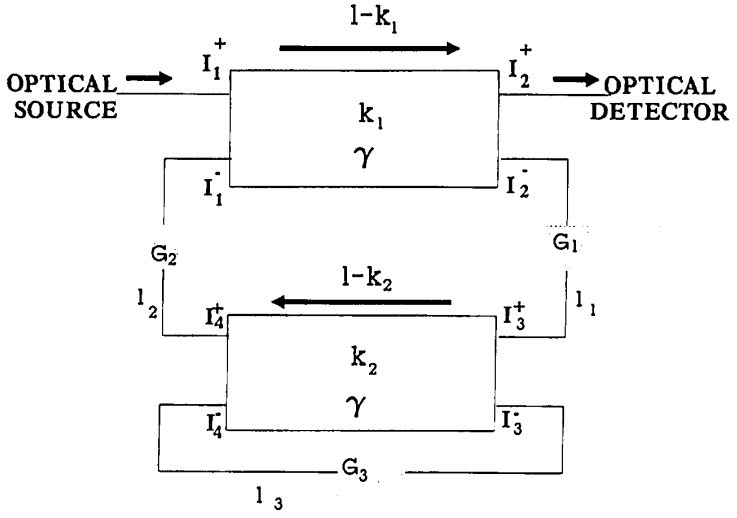

Fig. 1. Double-parallel amplified recirculating optical-delay line (DPAROD): $\quad \mathrm{I}_{j}^{\mathrm{s}}$ are the optical-field intensities at the input and output ports of the directional couplers, while $\gamma_{j}$ and $k_{j}$ are the fractional losses and the coupling ratio of the $j$ directional coupler.

sion is obtained:

$$
\begin{aligned}
H(\Omega)= & I_{2}{ }^{+} / I_{1}{ }^{+}=\left(1-\gamma_{1}\right)\left(1-k_{1}\right) \\
& +\left(1-\gamma_{1}\right) k_{1} \frac{A S_{1} S_{2}\left(1-\gamma_{1}\right) k_{1}}{1-\left(1-\gamma_{1}\right)\left(1-k_{1}\right) A S_{1} S_{2}}
\end{aligned}
$$

where

$$
\begin{aligned}
& S_{i}=G_{i} 10^{-\left(\alpha_{i} l_{\left.p_{i} / 10\right)} e^{j \Omega_{\tau_{i}}} \quad i=1,2,3\right.} \\
& A=\left(1-\gamma_{2}\right) \frac{\left(1-k_{2}\right)+\left(1-\gamma_{2}\right)\left(2 k_{2}-1\right) S_{3}}{1-\left[\left(1-\gamma_{2}\right)\left(1-k_{2}\right) S_{3}\right]} .
\end{aligned}
$$

The definitions of the utilized parameters are as follows: $\gamma_{j}, \alpha_{j}$, and $k_{j}$ are the coupler fractional losses, the fiber attenuation, and the coupling ratio of the directional couplers, respectively, as indicated in Fig. 1, where $j=1$ refers to coupler 1 and $j=2$ refers to coupler 2. $G_{1}, G_{2}$, and $G_{3}$ are the gains introduced in each of the fiber loop lines, and $\Omega$ is the frequency modulating the optical carrier. $l_{p_{i}}$ corresponds to the unamplified fiber length, while $\tau_{i}$ is the propagationtime delay introduced by each loop line $i$. These delays are given by the expression

$$
\tau_{i}=n\left(l_{p_{i}}+l\right) / c,
$$

where $l$ is the length of the amplified fiber, $c$ is the light speed in vacuum, and $n$ is the refractive index of the unamplified fiber (because the refractive-index difference between the amplified and the unamplified fiber is no greater than 0.02 , only one refractive index has been used).

The time delays given by Eq. (4) do not have to be integer multiples of a basic delay, so as explained above, the $Z$ transform cannot be applied.

The new method of analyzing DPAROD's consists of splitting the system into three single rings: The first is made of the fiber sections of length $l_{1}$ and $l_{2}$, as shown in Fig. 2(a). This loop is closed through coupler 2 , giving the consequent factor $\left(1-k_{2}\right)$ in the overall gain of the loop [see Eq. (6)]. The second ring contains the fiber of length $l_{3}$ [Fig. 2(b)]. The entire transfer function is multiplied by the factor $k_{1}^{2} G_{1} G_{2}$ because the first and the last optical field intensities cross through coupler 1 during each circulation.

The third ring comprises all three loop lengths $-l_{1}$, $l_{2}$, and $l_{3}$-and a factor of $\left(k_{2}\right)^{2}$ from crossing through coupler 2 is added [Fig. 2(c)] to the overall gain of the loop [see Eq. (8)].

Adding together the different results of the equations for the three rings yields an approximation of the output of the system. This method considers certain common terms, such as the direct pass through coupler 1 for rings 1,2 , and 3 or the direct pass across coupler 2 for rings 1 and 2 , only once.

The expression of the transfer function for each single ring is given by

$$
H(\Omega)=(1-\gamma)\left[\frac{(1-k)+M(2 k-1)}{1-M(1-k)}\right],
$$

where $M=G^{*} Z^{-1}$, with $G^{*}=10^{-(\alpha l p / 10)} \times G(1-\gamma)$,

(a)
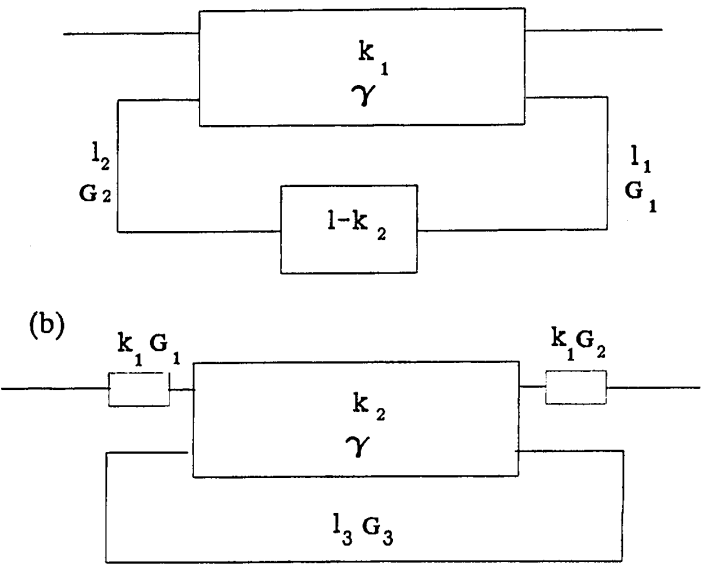

(c)

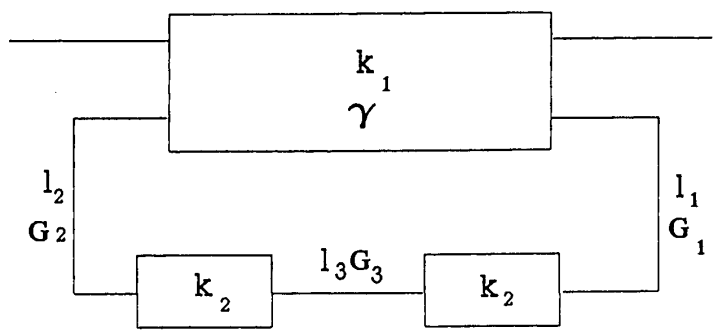

Fig. 2. Novel separation of the double structure into three different rings: (a) ring 1 comprises $l_{1}$ and $l_{2}$; (b) ring 2 comprises $l_{3} ;$ and (c) ring 3 comprises $l_{1}, l_{2}$, and $l_{3}$. 
$Z=e^{j \Omega \tau}$ and, for rings 1,2 , and 3 , respectively,

$$
\begin{array}{lll}
G=G_{1} G_{2}\left(1-k_{2}\right), & k=k_{1} ; \\
G=G_{3}, & k=k_{2} ; \\
G=G_{1} G_{2} G_{3} k_{2}^{2}, & k=k_{1} .
\end{array}
$$

The parameters that have not been identified a second time have the same meaning as in the doublering configuration.

For each single-ring configuration, it can be inferred from Eq. (5) that the frequencies $f$ of the maxima-minima of the transfer function are located at

$$
\begin{aligned}
& f_{\max 1}=L /\left(\tau_{1}+\tau_{2}\right), \\
& f_{\min 1}=(1 / 2+L) /\left(\tau_{1}+\tau_{2}\right) ; \\
& f_{\max 2}=M / \tau_{3}, \\
& f_{\min 2}=(1 / 2+M) / \tau_{3} ; \\
& f_{\max 3}=N /\left(\tau_{1}+\tau_{2}+\tau_{3}\right), \\
& f_{\min 3}=(1 / 2+N) /\left(\tau_{1}+\tau_{2}+\tau_{3}\right) .
\end{aligned}
$$

Here, $N, M$, and $L$ are integer numbers. These frequencies correspond to those of the laser-modulation frequency.

So all possible maximum and minimum frequencies belong to the set of values

$$
\begin{aligned}
f_{\max }= & L /\left(\tau_{1}+\tau_{2}\right) \cup M / \tau_{3} \cup N /\left(\tau_{1}+\tau_{2}+\tau_{3}\right) \\
f_{\min }= & (1 / 2+L) /\left(\tau_{1}+\tau_{2}\right) \cup(1 / 2+M) / \tau_{3} \cup(1 / 2+N) \\
& /\left(\tau_{1}+\tau_{2}+\tau_{3}\right) .
\end{aligned}
$$

From Eqs. (12) and (13) it can be inferred that the only parameters that influence the location of the maxima and the minima are $l_{1}, l_{2}, l_{3}$, and $n$. The relative amplitude of the different maxima and minima would be fixed through the remaining parameters $k_{1}$, $k_{2}, G_{1}, G_{2}$, and $G_{3}$.

\section{Periodicity of the Transfer Function}

Knowing that $H(\Omega)$ is a periodic function, it would be interesting to determine the margin of frequencies that represent one period of $H(\Omega)$. Considering again the division into three rings, the period of each separate ring would be $1 / f_{\max i}$, as shown in Eqs. (9)-(11). If rings 2 and 3 are considered simultaneously, the period of $H(\Omega)$ of the compound structure would be obtained by selecting a certain pair of the smallest integers $N$ and $M$, which follow

$$
N\left[1 /\left(\tau_{1}+\tau_{2}+\tau_{3}\right)\right]=M\left(1 / \tau_{3}\right),
$$

so $\mathrm{MFP}=M\left(1 / \tau_{3}\right)$, where MFP is the margin of frequencies of a period, which means the range in frequency between two consecutive main maxima.

Equation (14) is also valid when including ring 1, because if we operate with it

$$
N\left[1 /\left(\tau_{1}+\tau_{2}+\tau_{3}\right)\right]=M\left(1 / \tau_{3}\right)=L\left[1 /\left(\tau_{1}+\tau_{2}\right)\right] .
$$

\section{Other Terms Affecting the Model}

The general expression for the delay associated with this double configuration are as follows:

$$
\sum_{n=1}^{\infty} \sum_{m=1}^{\infty} \delta\left[t-n\left(\tau_{1}+\tau_{2}\right)-m\left(\tau_{3}\right)\right]
$$

So the transfer function would have a maximum at

$$
f_{\max }=\bigcup_{L=0}^{\infty} \bigcup_{N=0}^{\infty} \bigcup_{M=0}^{\infty} L /\left[N\left(\tau_{1}+\tau_{2}\right)+M\left(\tau_{3}\right)\right]
$$

This equation could imply that Eq. (12) was incomplete, but that conclusion would be wrong because the new terms added in Eq. (15) would never contribute to generate a maximum frequency. Let us consider, for example, the initial second-order term, which has been neglected: $M=1$ and $N=2$, indicating one trip around ring 3 and two trips around ring 1 . It can be seen that, although completion of the total path can cause in-phase interference, other interference at the $\mathrm{I}_{2}{ }^{+}$terminal have occurred during the intermediate steps (see Fig. 1), and the interference may have canceled the effects at $I_{2}{ }^{+}$. The same reasoning can be applied to the minima frequencies.

\section{Overlap Effect}

For a certain set of values $\left(k_{1}, k_{2}, G_{1}, G_{2}\right.$, and $\left.G_{3}\right)$, in which the influence of the three rings is fairly similar, an overlap effect would appear such that the frequencies of the secondary maxima would suffer a shift with respect to the habitual case, where overlap is not present. This overlap usually occurs when the coupling coefficients $k_{1}$ and $k_{2}$ are approximately 0.5 , which we do not consider useful for our purposes. An example where this effect appears follows: The parameters of a structure where overlap could occur are $k_{1}=0.72, k_{2}=0.63, G_{1}=G_{2}=G_{3}=1, n=1.44$, $l_{1}=1 \mathrm{~m}, l_{2}=2 \mathrm{~m}, l_{3}=3 \mathrm{~m}, \alpha=0$, and $\gamma=0$. Table 1 lists the frequencies of the maxima determined with the simulation. ${ }^{1}$ The second column gives the results calculated with Eq. (12), as if no overlap effect existed.

Table 1. Calculated and Simulated Frequencies ${ }^{a}$

\begin{tabular}{cc}
\hline Simulated $f_{\max }(\mathrm{MHz})$ & Calculated $^{b} f_{\max }(\mathrm{MHz})$ \\
\hline Frequencies & \\
0 & 0 \\
45.37 & 41.66 \\
84.42 & 83.33 \\
123.48 & 125 \\
163.11 & 166.66 \\
208.33 & 208.33 \\
\hline
\end{tabular}

${ }^{a}$ Parameters are $k_{1}=0.72, k_{2}=0.63, G_{1}=G_{2}=G_{3}=1, n=1.44$, $l_{1}=1 \mathrm{~m}, l_{2}=2 \mathrm{~m}, l_{3}=3 \mathrm{~m}, \alpha=0$, and $\gamma=0$.

${ }^{b}$ Frequencies calculated with Eq. (12). 
Table 2. Calculated and Measured Frequencies for Maxima and Minima ${ }^{a}$

\begin{tabular}{|c|c|c|c|c|c|}
\hline \multicolumn{2}{|c|}{ Calculated $f_{\max }^{b}$} & \multicolumn{2}{|c|}{ Calculated $f_{\min }$} & \multicolumn{2}{|c|}{$\begin{array}{c}\text { Measured } \\
\text { Frequencies }^{c}\end{array}$} \\
\hline $\begin{array}{c}\text { Contributing } \\
\text { Ring }\end{array}$ & $\mathrm{MHz}$ & $\begin{array}{c}\text { Contributing } \\
\text { Ring }\end{array}$ & $\mathrm{MHz}$ & $\begin{array}{c}f_{\max } \\
(\mathrm{MHz}) \\
\end{array}$ & $\begin{array}{c}f_{\min } \\
(\mathrm{MHz})\end{array}$ \\
\hline & & $\begin{array}{l}3 \\
1\end{array}$ & $\begin{array}{l}17.36 \\
26.04\end{array}$ & & \\
\hline 3 & 34.7 & & & 34.4 & \\
\hline $\begin{array}{l}1 \\
3\end{array}$ & $\begin{array}{l}52.08 \\
69.4\end{array}$ & 3,2 & 52.06 & 69.3 & 52.04 \\
\hline & & $\begin{array}{l}1 \\
3\end{array}$ & $\begin{array}{l}78.12 \\
86.76\end{array}$ & & 86.0 \\
\hline $1,2,3$ & 104.1 & $\begin{array}{l}3 \\
1\end{array}$ & $\begin{array}{l}121.46 \\
130.2\end{array}$ & 103.5 & 120.9 \\
\hline 3 & 138.8 & & & 139.2 & \\
\hline $\begin{array}{l}1 \\
3\end{array}$ & $\begin{array}{l}156.18 \\
173.5\end{array}$ & 2,3 & 156.115 & 173 & 155.2 \\
\hline & & $\begin{array}{l}1 \\
3\end{array}$ & $\begin{array}{l}182.58 \\
190.82\end{array}$ & & 190.2 \\
\hline $1,2,3$ & 208.26 & & & 207.8 & \\
\hline
\end{tabular}

${ }^{a}$ Frequencies where maxima and minima can be located when $l_{1}=l_{2}=l_{3}=2 \mathrm{~m}$ and $n=1.44$.

${ }^{b}$ Calculated $f_{\max }$ determined with Eq. (12); calculated $f_{\min }$ determined with Eq. (13).

${ }^{c}$ Maxima and minima measured when $l_{1}=l_{2}=l_{3}=2 \mathrm{~m}, \alpha_{1}=$ $\alpha_{2}=\alpha_{3}=0.0003 \mathrm{~dB} / \mathrm{m}, \gamma_{1}=\gamma_{2}=0.05, k_{1}=0.958, k_{2}=0.912$, $G_{1}=G_{3}=0.5$, and $G_{2}=0.25$.
Table 3. Prevailing Rings as $k_{1}$ and $k_{2}$ Are Varied ${ }^{a}$

\begin{tabular}{ccc}
\hline & \multicolumn{2}{c}{ Prevailing Ring } \\
\cline { 2 - 3 } Conditions & $k_{1} \Rightarrow 1$ & $k_{1} \Rightarrow 0$ \\
\hline$k_{2} \Rightarrow 1$ & 2 & 3 \\
$k_{2} \Rightarrow 0$ & $1,2^{b}$ & 1 \\
\hline
\end{tabular}

${ }^{a}$ All other DPAROD parameters are kept constant.

${ }^{b}$ The influence of ring 2 increases as $k_{1}$ approaches unity.

\section{Experimental Confirmation}

A cw laser-diode output of $1.5 \mu \mathrm{m}$ was sinusoidally modulated in frequency by using the tracking generator of an rf spectrum analyzer at the desired frequencies. This modulated signal was the input to the recirculating delay line. The set up was constructed with two $2 \times 2, k$-variable, polarization-preserving fiber couplers with $2 \mathrm{~m}$ of pigtails that constitute the different recirculating delay lines of the DPAROD, that is $l_{1}=l_{2}=l_{3}=2 \mathrm{~m}$. According to Eqs. (12) and (13), it is possible for maxima and minima to be located at the frequencies listed in the second and third columns of Table 2 . It is important to note that these are the frequencies where the maxima can be located, but choosing values of some parameters (e.g., $k_{1}, k_{2}, G_{1}, G_{2}$, and $G_{3}$ ), permits the amplitude of the optical field to be low enough that there is not a maximum at some of those frequencies. This can be the case with the minimum frequencies. In Table 2, the first and fourth columns list the ring or rings that contribute to the maximum and the minimum fre-

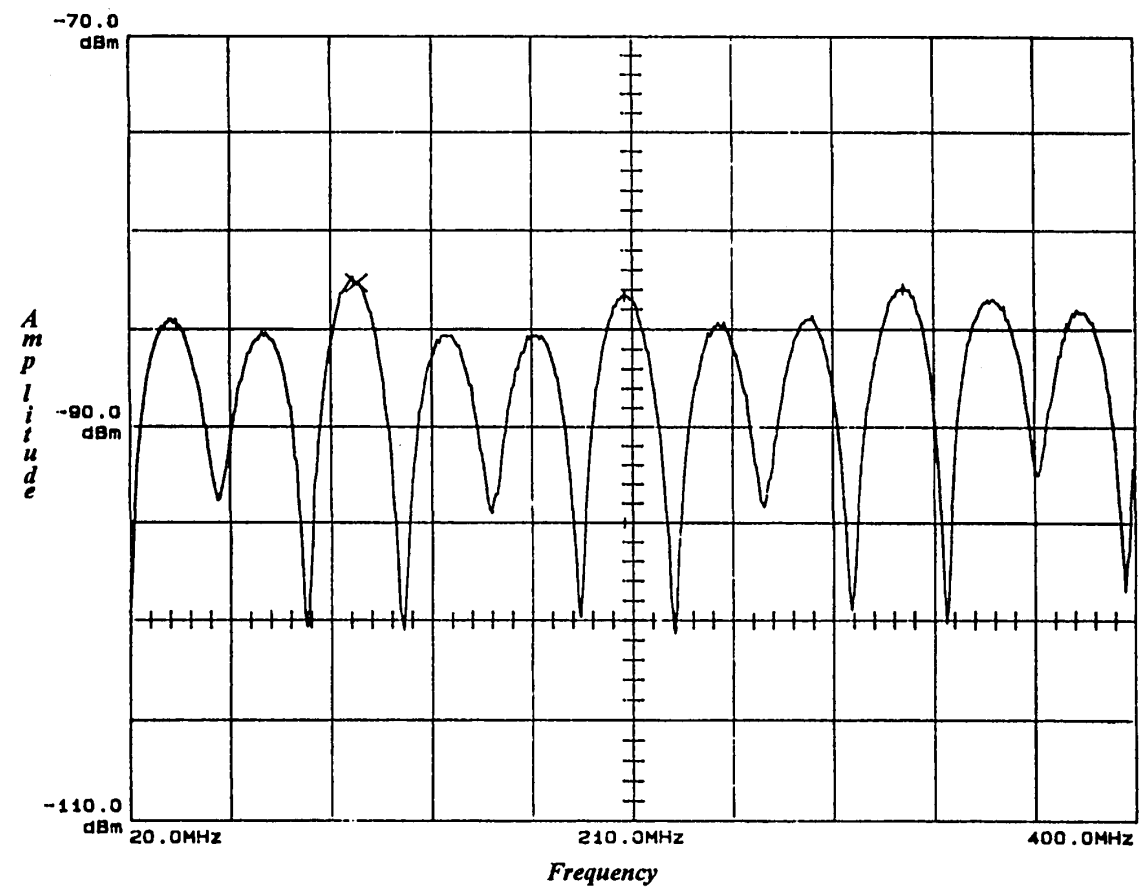

Fig. 3. Transfer function of the DPAROD (experimental results) for $l_{1}=l_{2}=l_{3}=2 \mathrm{~m}, \alpha_{1}=\alpha_{2}=\alpha_{3}=0.0003 \mathrm{~dB} / \mathrm{m}, \gamma_{1}=\gamma_{2}=0.05, n=$ $1.44, k_{1}=0.958, k_{2}=0.912, G_{1}=G_{3}=0.5$, and $G_{2}=0.25$. 

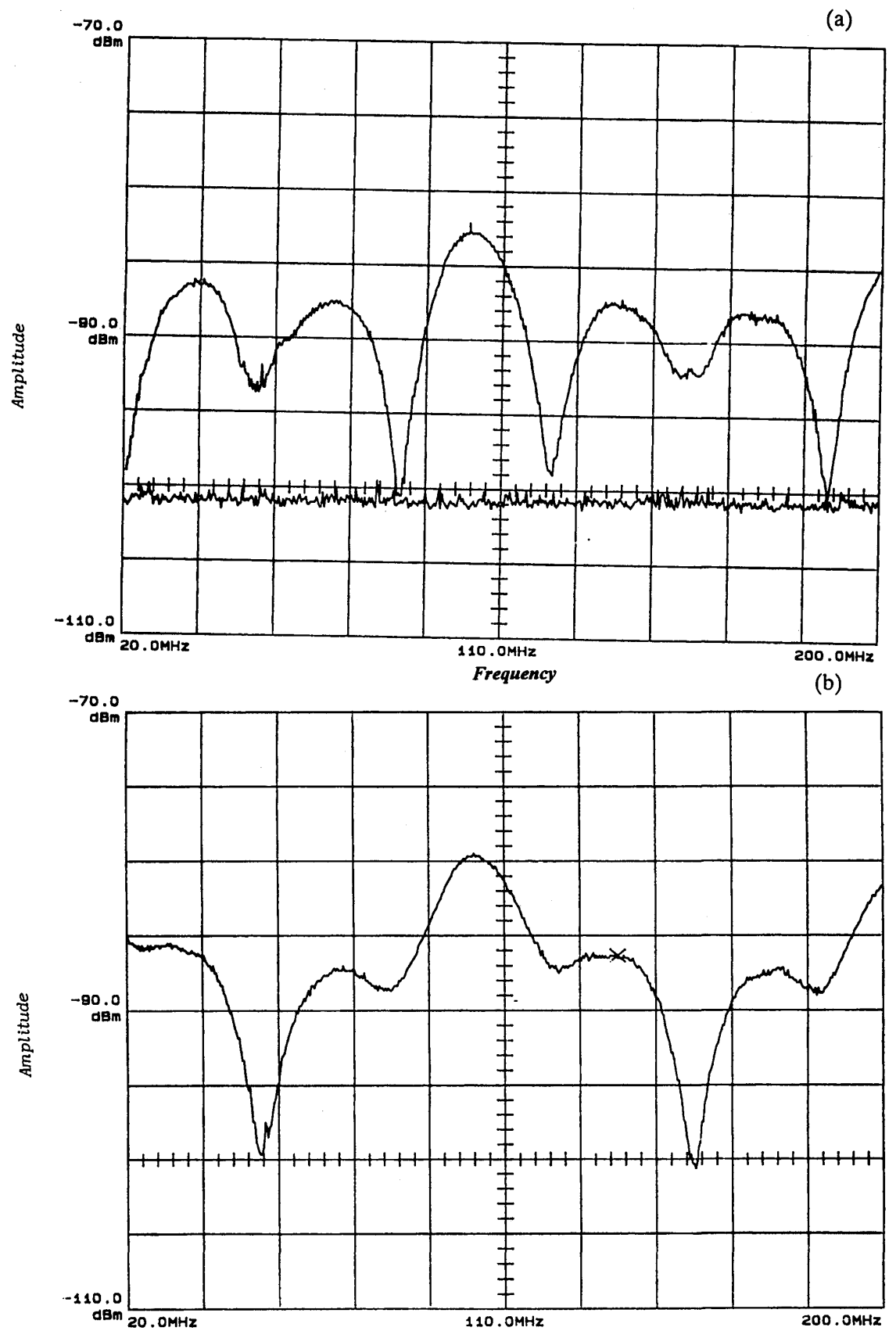

Fig. 4. Transfer function of the DPAROD (experimental results) for $l_{1}=l_{2}=l_{3}=2 \mathrm{~m}, \alpha_{1}=\alpha_{2}=\alpha_{3}=0.0003 \mathrm{~dB} / \mathrm{m}, \gamma_{1}=\gamma_{2}=0.05, n=$ 1.44, $G_{1}=G_{3}=0.5, G_{2}=0.25$, and $k_{2} 0.86: \quad \mathrm{a}, k_{1}=0.95$; and b, $k_{1}=0.98$.

quencies, respectively, and the fifth and sixth columns list the measured frequencies for $k_{1}=0.958$, $k_{2}=0.912, G_{1}=G_{3}=0.5, G_{2}=0.24, \alpha=0.03$, and $\gamma=0.05$ (see Fig. 3). Through this example, which shows the periodicity of $H(\Omega)$, we can analyze one MFP of the transfer function. The absolute maxima are those where the three rings contribute to achieve the maximum frequency $(103.5 \mathrm{MHz})$. The other frequencies correspond to the minima of ring 3 (at 120.9 and $190.2 \mathrm{MHz}$ ) or to the maxima of the same ring. It can be seen that, for those frequencies where the maxima and the minima of different rings overlap $\left(f_{\max 1}=f_{\min 2,3}=52 \mathrm{MHz}\right)$ because of the great influence of rings 2 and 3 (see the next section and 


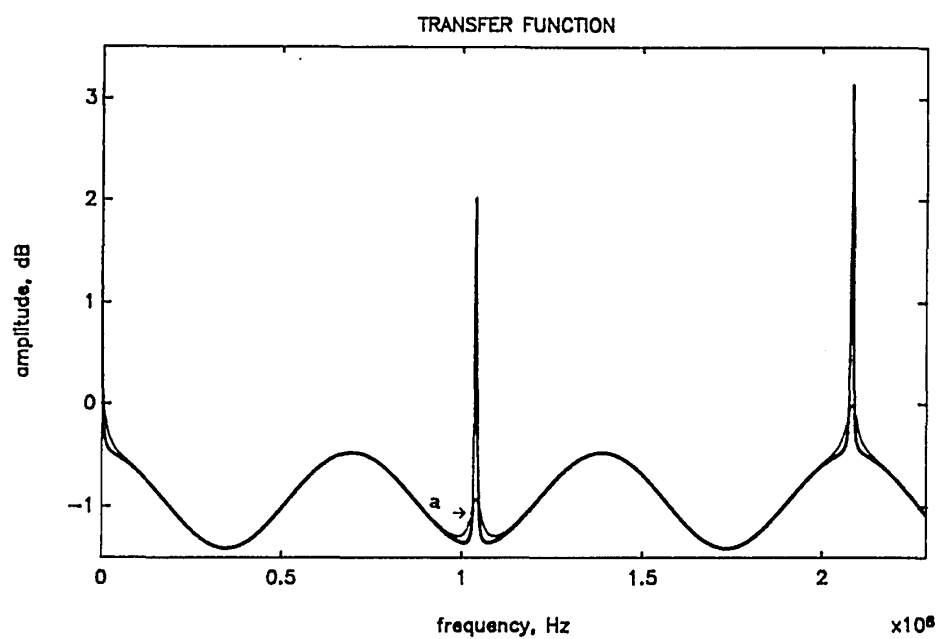

Fig. 5. Transfer function of the DPAROD (calculated) for $k_{1}=0.95, k_{2} 0.1, L_{1}=1 \mathrm{~m}, L_{2}=L_{3}=2 \mathrm{~m}, n=1.44, \alpha=\gamma=0$, and $G_{1}=G_{2}=1$ : a, $G_{3}=1 ; b, G_{3}=1.1$.

Table 3), the measured amplitude corresponds to a minimum. If other lengths were selected, the FSR would increase, as in Ref. 5 . With respect to the MFP, by applying Eq. (15) we find that $104.1 M=$ $52.08 L$, so $L / M=2$, which means that for $L=2$ and $M=1$, the margin frequency $=104.1 \mathrm{MHz}$, as can be seen in Fig. 3 and Table 2. There is a perfect agreement between the measured and predicted values, so the model is demonstrated to be valid for determining the maxima and the minima.

\section{Influence of the Different Parameters on the Frequency Response}

Throughout this analysis, the parameters that are not being tested will remain constant at $G_{i}=1, \alpha_{i}=0$, and $\gamma_{i}=0$.

\section{Influence of the Refractive Index and of the Loop Lengths}

The refractive index $n$ and the lengths of the fiber loops $l_{1}, l_{2}$, and $l_{3}$ are the parameters that determine the location of the maxima. The effect of varying any of them would be similar. A significant case is when $l_{3}=l_{1}+l_{2}$, so the maxima of rings 1 and 2 are superimposed, and a secondary maximum will appear only at the half of the main one. If any of the loop lengths are changed, more secondary maxima will appear.

\section{Influence of $k_{1}$ and $k_{2}$}

As a first step it is necessary to delimit the ranges of $k_{1}$ and $k_{2}$ to those where the response is governed by a single ring. By comparing any two rings (e.g., $i$ and $j$ ), the ratio $H(\Omega)_{\max _{i}} / H(\Omega)_{\max _{j}}$ is calculated. By changing $k_{1}$ and $k_{2}$, this ratio can be increased or decreased, showing the prevailing ring or rings in each case. If only one ring dominates, the analysis of the structure will be equivalent to that of a single ring $^{2}$ with an equivalent gain, which would be de- scribed by the expressions in Eqs. (6)-(8). The expressions of the different ratios are omitted and only the conclusions are given in Table 3 . This analysis is also applicable to the minima.

It can be seen in Fig. 4 how, at 86.0 and $120.9 \mathrm{MHz}$, there are two minima whose levels increase when $k_{2}=0.86$ and $k_{1}$ changes from 0.95 [Fig. $4(\mathrm{a})$ ] to 0.98 [Fig. 4(b)]. As $k_{1}$ changes, the influence of ring 2 increases with respect to ring 3 , as predicted in Table 3 (see conditions for $k_{1} \Rightarrow 0$, and $k_{1} \Rightarrow 1$ when $k_{2} \Rightarrow 1$ ). The same effects occur at 155.2 and 52.0 $\mathrm{MHz}$, which decrease because they correspond to the minima of ring 2 . The rest of the parameters of the structure have been kept unaltered, i.e., $G_{1}=G_{3}=$ $0.5, G_{2}=0.25, \alpha=0.03 \mathrm{~dB} / \mathrm{km}, \gamma=0.05, l_{1}=l_{2}=$ $l_{3}=2 \mathrm{~m}$. This example helps us to understand the utility of Table 3 and its possible interpretation.

Influence of $G_{1}, G_{2}$, and $G_{3}$

Following the same steps as in the preceding section and taking into account the gains $G_{i}$ of each loop, the results shown in Table 3 still prevail, but more degrees of freedom $\left(G_{1} \times G_{2}, G_{3}\right)$ have been added to the system. Now the new gains will be determined by Eqs. (6)-(8) by allowing the values of $G_{1}, G_{2}$, and $G_{3}$ to change.

Special attention is given to the influence of $G_{3}$ when $k_{2} \Rightarrow 0$. From Table 2 we determine that if $k_{2} \Rightarrow 0$ and $k_{1} \Rightarrow 1$, the influence of both ring 1 and ring 2 appears. But changes in $G_{3}$ affect only ring 2 . This is confirmed in Fig. 5, where $k_{1}=0.95$ and $k_{2}=$ $0.1\left(l_{1}=1 \mathrm{~m}, l_{2}=2 \mathrm{~m}, l_{3}=2 \mathrm{~m}, n=1.44, \alpha=\gamma=0\right.$, $G_{1}=G_{2}=1$ ) and $G_{3}$ changes from 1 to 1.1 (linear scale) so the amplitude of the maximum at $104.2 \mathrm{MHz}$ (related to ring 2) increases while the maximum of ring 1 remains the same. So only the amplitude for a fixed frequency has been altered, and the rest of the transfer function has not been modified. This behav- 
ior is different from that of a typical tuner, where all elements in the transfer function are shifted.

Influence of the Attenuation of the Fiber $\alpha$

Any change in $\alpha$ is equivalent to a change in the gain loop, because it can be defined as

$$
G_{i}^{*}=G_{i} \times 10^{-\alpha l p i / 10} .
$$

Influence of Fractional Losses at the Couplers

Losses at the couplers can be taken into account when considering the factor $\left(1-\gamma_{1}\right)$ related to each coupler and should be included into the overall gain:

$$
G_{i}{ }^{\prime}=\left(1-\gamma_{i}\right) G_{i}^{*} .
$$

\section{Summary}

Determining the locations of the maximum and minimum frequencies in double-parallel amplified recirculating optical delay lines (DPAROD), while essential for their application as filters, cannot be obtained using the $Z$ transform techniques, as can be done for single amplified recirculating delay lines. In this work, we have presented a new model for calculation of these maximum and minimum frequencies. The model consists of splitting the whole system into three independent rings. Experimental results have been reported to help to understand its meaning.

The versatility of DPAROD's has been shown, and the model has been applied to the analysis of the influence of different parameters on the shape of the transfer function. The model permits one to delimit the intervals for the values of the different parameters that should be interesting to work in filtering (or demultiplexing) applications.

This work was supported by Spanish Comunidad Autónoma de Madrid-contract PRI (C059/90), Comision Inteerministerial de Ciencia y Tecnologia TIC920052-C02 and 89-211, and Universidad Politécnica de Madrid (Acciones Concertadas). We thank B. Vizoso and ATT España S.A. for their help.

\section{References}

1. M. C. Vázquez, B. Vizoso, M. López-Amo, and M. A. Muriel, "Single and double recirculating delay lines as fiber-optic filters," Electron. Lett. 28, 1017-1019 (1992).

2. B. Mosheli and J. W. Goodman, "Novel amplified fiber-optic recirculating delay line processor," J. Lightwave Technol. 10, 1142-1147 (1992).

3. K. P. Jackson, S. A. Newton, B. Moslehi, M. Tur, C. Chapin Cutler, J. W. Goodman, and H. J. Shaw, "Optical fiber delayline signal processing," IEEE Trans. Microwave Theory Tech. 33, 193-209 (1985).

4. T. J. Soukup, R. J. Feuerstein, and V. P. Heuring. "Implementation of a fiber-optic delay-line memory," Appl. Opt. 31, 32333240 (1992).

5. K. Oda, N. Takato, and H. Toba, "A wide FSR waveguide double ring resonator for optical FDM transmission systems," J. Lightwave Technol. 9, 728-736 (1991).

6. B. Mosheli, J. W. Goodman, M. Tur, and H. J. Shaw, "Fiberoptic lattice signal processing," IEEE Proc. Natl. Aerosp. Electron. Conf. 72, 909-930 (1984). 